\title{
Repetitive spinal motor neuron discharges following single transcranial magnetic stimulation: relation to dexterity
}

\author{
W. J. Z'Graggen • A. M. Humm • \\ S. Oppliger-Bachmann $\cdot$ M. Hosang $\cdot$ K. M. Rösler
}

Received: 20 February 2008 / Accepted: 13 April 2008 / Published online: 8 May 2008

(C) Springer-Verlag 2008

\begin{abstract}
Transcranial magnetic stimulation allows to study the properties of the human corticospinal tract noninvasively. After a single transcranial magnetic stimulus, spinal motor neurons (MNs) sometimes fire not just once, but repetitively. The biological significance of such repetitive MN discharges (repMNDs) is unknown. To study the relation of repMNDs to other measures of cortico-muscular excitability and to physiological measures of the skill for finely tuned precision movements, we used a previously described quadruple stimulation (QuadS) technique (Z'Graggen et al. 2005) to quantify the amount of repMNDs in abductor digiti minimi muscles (ADMs) on both sides of 20 right-handed healthy subjects. Skillfulness for finger precision movements of both hands was assessed using a finger tapping task. In 16 subjects, a follow-up examination was performed after training of either precision movements $(n=8)$ or force $(n=8)$ of the left ADM. The size of the QuadS response (amplitude and area ratios) was greater in the dominant right hand than in the left hand (QuadS amplitude ratio: $47.1 \pm 18.1$ versus $37.7 \pm 22.0 \%$, Wilcoxon test: $P<0.05$; QuadS area ratio: $49.7 \pm 16.2 \%$ versus $36.9 \pm 23.0 \%$, Wilcoxon test: $P<0.05$ ), pointing to a greater amount of repMNDs. Moreover, the QuadS amplitude and area increased significantly after finger precision training, but not after force training. This increase of repMNDs correlated significantly with the increase in performance in the finger tapping task. Our results demonstrate that repMNDs are related to handedness and therefore
\end{abstract}

W. J. Z'Graggen ( $₫) \cdot$ A. M. Humm · S. Oppliger-Bachmann ·

M. Hosang · K. M. Rösler

Department of Neurology, Inselspital,

Bern University Hospital and University of Bern,

3010 Bern, Switzerland

e-mail: werner.zgraggen@insel.ch probably reflect supraspinal excitability differences. The increase of repMNDs after skills training but not after force training supports the hypothesis of a supraspinal origin of repMNDs.

Keywords Transcranial magnetic stimulation . Collision technique $\cdot$ Corticospinal tract plasticity

\section{Introduction}

After a single magnetic brain stimulus, spinal motor neurons (MNs) may discharge more than once (Berardelli et al. 1991; Day et al. 1987; Hess et al. 1987; Naka and Mills 2000). We recently described a new method to assess repetitive MN discharges (repMNDs), which combines the triple stimulation technique (TST) with an additional nerve stimulus in the periphery (quadruple stimulation, QuadS) (Z'Graggen et al. 2005). The QuadS eliminates the first action potential descending on each axon after TMS and eliminates effects on response size induced by desynchronization of these discharges, thereby allowing a quantification of MNs discharging twice.

The biological significance of these repMNDs is unknown. Moreover, the amount of repMNDs is probably influenced both at the spinal segmental level as well as supraspinally, but the contribution of either mechanism is also unclear (Z'Graggen et al. 2005). It has been shown that motor evoked potentials (MEPs) elicited by stimulation of the dominant hemisphere are often larger than those after stimulation of the non-dominant hemisphere (Brouwer et al. 2001; De Gennaro et al. 2004; Netz et al. 1995) and that cortical representation of the dominant hand is larger than the one of the non-dominant hand (Cantello et al. 1992; Krings et al. 1997; Triggs et al. 1999). This asymmetry of 
the motor cortex seems not to be associated with functional differences in callosal inhibition (De Gennaro et al. 2004; Civardi et al. 2000). Furthermore, the size of MEPs and the cortical area of representation increase, whereas the threshold for MEPs decreases after training of precision movements (Classen et al. 1998; Muellbacher et al. 2001; Pascual-Leone et al. 1995; Perez et al. 2004). Up to now, the underlying mechanisms of the size dependence of MEPs to handedness and training are not fully understood. In particular, it is not clear if differences in the amount of repMNDs participate to produce this side difference.

It was the aim of this study to elucidate the effect of handedness and motor training on the amount of single and repMNDs in a small hand muscle. A difference of repMNDs related to handedness would point to supraspinal and probably cortical excitability differences involved in the generation of repMNDs. Training of precision movements induces cortical adaptations; hence, an increase of repMNDs to training skills would also support a role of cortical excitability for repMNDs. In contrast, training of muscular force changes the functional properties of spinal cord circuitry (along with the muscle hypertrophy) without affecting the organization of the primary motor cortex (Carroll et al. 2002). Hence, if force training led to an increased number of repMNDs, spinal segmental mechanisms could account for it.

We used the triple and QuadS techniques to quantify the amount of MNs responding once or twice to a brain stimulus (Magistris et al. 1998; Z'Graggen et al. 2005). Our study demonstrates that, indeed, repMNDs are increased in the dominant hand and after training of precision movements, but not after force training.

\section{Methods}

Subjects and ethical approval

Twenty healthy subjects participated in this study (11 women and 9 men) after giving written informed consent. Their mean age was 24.1 years (range 22-28 years). All subjects were right-handed according to the Edinburgh Inventory of Handedness (Oldfield 1971). All procedures were approved by the local ethics committee (Kantonale Ethikkommission Bern) and conformed to the Declaration of Helsinki.

\section{Electrophysiology}

\section{EMG and force recordings}

Subjects were comfortably seated in a chair. Forearm, hand, and fingers II to IV were immobilized in a splint. EMG recordings were obtained from the abductor digiti minimi muscle (ADM) with silver surface electrodes (diameter
$0.8 \mathrm{~cm}$ ) in a tendon belly montage. The ground electrode was placed at the wrist. Measurements were made using a Viking Select apparatus (Nicolet, Madison, WI, USA). Bandpass filters were $2-10 \mathrm{kHz}$. The isometric voluntary contraction force of finger $\mathrm{V}$ abduction was measured by placing the finger against a lever attached to a force transducer (Sensotec Inc., OH, USA). The force signal was DC amplified using a Sedia amplifier (Sedia, Givisiez, Switzerland) and digitalized at $4 \mathrm{kHz}$ by a stand alone AD converter (MacLab, ADInstruments Pty Ltd., Castle Hill, NSW, Australia) connected to a personal computer. During the experiments, visual feedback of the exerted force was given by displaying the force signal on a computer screen in front of the subjects (Arányi et al. 1998; Rösler et al. 2002). After assessment of maximal voluntary abduction force (MVC), the target force level for facilitation was set to $20 \%$ of MVC and was indicated as goal marked on the screen.

\section{Peripheral nerve stimulation}

The ulnar nerve was stimulated supramaximally at the wrist (yielding the $\left.\mathrm{CMAP}_{\text {wrist }}\right)$ and at Erb's point $\left(\mathrm{CMAP}_{\mathrm{Erb}}\right)$, using a monopolar stimulation method (Magistris et al. 1998; Roth and Magistris 1987). Compound muscle action potentials (CMAPs) of the ADM were recorded at rest and during a contraction of $20 \%$ MVC. To access the excitability of the spinal MN pool, areas of ulnar F-waves were measured following 30 wrist stimuli and expressed as mean area ratio (F-wave: $\left.\mathrm{CMAP}_{\text {wrist }}\right)$.

\section{Transcranial magnetic stimulation}

Motor evoked potentials (MEPs) were obtained using a Magstim 200 (Magstim Company, Spring Gardens, Withland, Dyfed, UK) with a circular $90 \mathrm{~mm}$ hand-held coil. The intensity of the TMS pulse was expressed as a percentage of the maximal output of 2.0 Tesla. The center of the coil was placed over the vertex or slightly lateral toward the target hemisphere with the coil current flowing clockwise (when viewed from above) to excite the right hemisphere and anticlockwise to excite the left hemisphere. Small displacements were made in all directions until the position yielding the lowest threshold to stimulate the contralateral ADM was found. Resting motor threshold (RMT) was defined as the lowest TMS intensity that elicited MEPs of at least $50 \mu \mathrm{V}$ peak-to-peak amplitude in 5 or more out of 10 consecutive trials at rest (Conforto et al. 2004; Rossini et al. 1994). The intensity of TMS was then set to $150 \%$ of RMT for all recordings. MEP recordings were obtained during a background ADM contraction of $20 \%$ MVC. For analysis, mean MEP peak-to-peak amplitude and mean MEP area (mean of four trials), expressed as amplitude and area

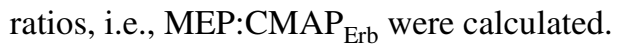




\section{Triple stimulation technique}

The TST is a collision method using a sequence of three stimuli, to the brain, the ulnar nerve at the wrist, and the brachial plexus at Erb's point (Magistris et al. 1998, 1999). The $\mathrm{TST}_{\text {test }}$ response is calibrated by a $\mathrm{TST}_{\text {control }}$ response, for which the brain stimulus is replaced by a stimulus at Erb's point (succession of stimuli: Erb-wrist-Erb). The TST eliminates both the effects of desynchronization of the TMS-induced MN discharges, and the effects of repMNDs (see Fig. 1a, b for a summary of the principle of the technique). The timing of the three stimuli was achieved by using a dedicated software package for the Nicolet Viking apparatus provided by Judex AS (Aalborg, DK). The delays between the three stimuli were calculated as follows:

- delay $\mathrm{I}=$ minimal MEP latency $-\mathrm{CMAP}_{\text {wrist }}$ latency

- delay II $=\mathrm{CMAP}_{\mathrm{Erb}}$ latency $-\mathrm{CMAP}_{\text {wrist }}$ latency .

The delays for the $\mathrm{TST}_{\text {control }}$ recording were calculated as follows:

- delay $\mathrm{I}=$ delay $\quad \mathrm{II}=\mathrm{CMAP}_{\mathrm{Erb}} \quad$ latency $-\mathrm{CMAP}_{\text {wrist }}$ latency.

Recordings were always performed during a background ADM contraction of $20 \%$ MVC. The proportion of spinal MNs excited by TMS was measured by the TST amplitude and area (mean of three trials), expressed as amplitude, and area ratios, i.e., $\mathrm{TST}_{\text {test }}: \mathrm{TST}_{\text {control }}$ (Magistris et al. 1998).

\section{Quantification of repMNDs: QuadS}

The principles of the QuadS were previously described in detail (Z'Graggen et al. 2005) (see Fig. 1c, d for a summary of the principle of the technique). As an addition to the TST, a fourth stimulus was given at the wrist, using an external electrical stimulator (Digitimer D185, Digitimer Ltd., Welwyn Garden City, Hertfordshire, England). The sequence of stimuli was: brain-wrist 1-wrist 2-Erb. The delays of brain-wrist 1-Erb were the same as those used for the $\mathrm{TST}_{\text {test }}$. The interstimulus interval between wrist 1 and wrist 2 was $3 \mathrm{~ms}$. By comparing it to the TST control curve (Fig. 1b), the percentage of MNs discharging twice in response to the brain stimulus was calculated. Recordings were made during a faciliatory ADM contraction of $20 \% \mathrm{MVC}$. The percentage of MNs with repMNDs was calculated by the QuadS amplitude and area ratio (QuadS test $:$ TST $\left._{\text {control }}\right)$ (Z'Graggen et al. 2005).

\section{Assessment precision movements of digit $\mathrm{V}$}

Subjects were comfortably seated in a chair with the forearm, hand, and fingers II to IV fixed on a table. They were asked to perform graded abductions and adductions of digit $\mathrm{V}$ by tapping repetitively on three on a pattern premarked positions (maximal adduction $\rightarrow$ middle position between maximal abduction and maximal adduction $\rightarrow$ maximal abduction $\rightarrow$ middle position $\rightarrow$ maximal adduction). The number of correctly performed digit movements (defined as taps that did not deviate by more than $50 \%$ digit width from the premarked position) was counted during $15 \mathrm{~s}$ in three consecutive trials, with a break of $2 \mathrm{~min}$ between each block to minimize fatigue. The mean number of correct taps of three trials was assessed.

Experimental protocol and training

Both hands of each subject were examined. The side examined first was chosen randomly. One experimental session consisted of assessment of the precision movements and electrophysiological testing as described earlier. In each subject, 4 MEP curves, $3 \mathrm{TST}_{\text {test }}$ curves, $1 \mathrm{TST}_{\text {control }}$ curve, 3 QuadS $_{\text {test }}$ curves, and 1 QuadS $_{\text {control }}$ curve were recorded. In a subgroup of 16 subjects, the same experimental protocol was repeated 7 days later after either a training of the precision abduction task $(n=8)$ or a training of ADM force $(n=8)$. The training modality was assigned randomly to each of the subjects, and only the left hand was trained. Subjects started the training at the day following the first examination. They were asked to perform two training sessions per day of 5 min duration each (one in the morning, one in the evening). The last training session was $24 \mathrm{~h}$ before the re-examination. Training of the precision abduction task was practiced by performing stepwise precision abductions and adductions as described earlier using the same premarked pattern. Force training was performed by maximal isometric abduction of digit $\mathrm{V}$ against resistance with the wrist in neutral position.

\section{Analysis}

All statistical analyses were performed using SPSS 14.0 (SPSS Inc., USA). Pretraining results of both hands (RMT, MEP amplitude and area ratios, TST amplitude and area ratios, QuadS amplitude and area ratios, and mean number of correct finger taps during $15 \mathrm{~s}$ ) were compared using Wilcoxon test. To analyze the effects of training on these parameters, differences of the values after training-values before training were calculated. Training effects were compared using Kruskal-Wallis test.

\section{Results}

Assessment before training

RMT, MEP, and TST responses

Average RMT was 37.3\% (SD 5.3\%) for the left and 38.8\% (4.9\%) for the right primary motor cortex $(P>0.05)$. Mean 
(A) $\mathrm{TST}_{\text {test }}$

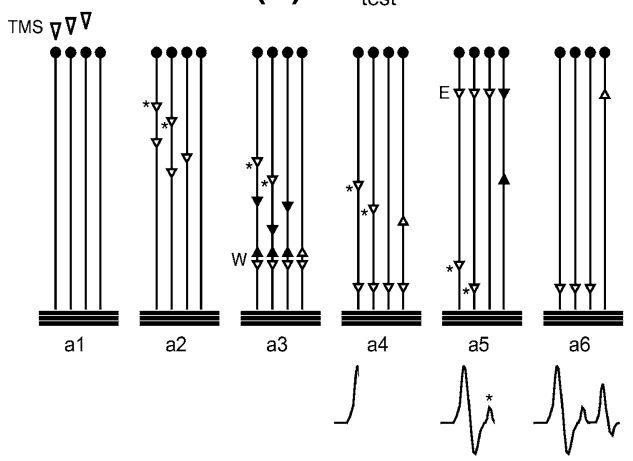

(C) QuadS $_{\text {test }}$

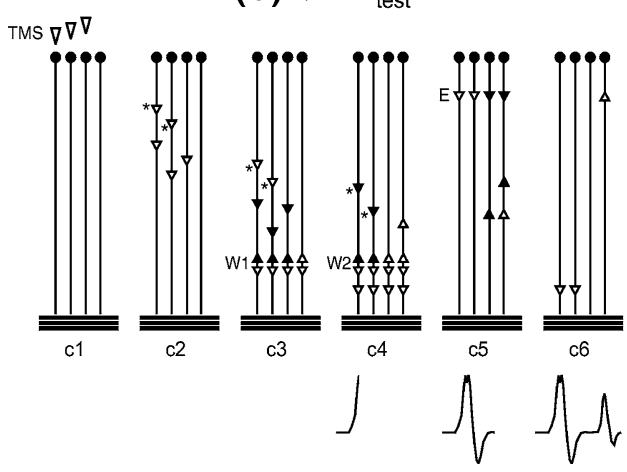

Fig. 1 a Triple stimulation technique (TST) principle. The peripheral motor tract is simplified to 4 spinal motor neurons (MNs); horizontal lines represent the muscle fibers of the 4 motor units. Black arrows represent action potentials that collide; arrows with an asterisk mark, repMNDs. a In $\mathrm{TST}_{\text {test }}: a 1$ a submaximal transcranial stimulus excites 3 spinal MNs of 4 (open arrows). a2 On 3 of 4 MNs, TMS induced action potentials descend. Desynchronization of the 3 action potentials has occurred (possibly at spinal cell level), 2/3 spinal MNs are excited twice causing repMNDs. a3 After a delay, a maximal stimulus is applied at the wrist (W). $a 4$ It gives rise to a first main negative deflection of the recording trace. The antidromic action potentials collide with the first descending action potentials on MNs 1, 2, and 3. The repMND on MNs 1 and 2 do not collide and give rise to a small negative deflection of the recording trace (marked by $*$ in $a 5$ ). The action potential on MN 4 continues to ascend. $a 5$ After an appropriate delay, a maximal stimulus is applied at Erb's point (E). On MN 4, the descending action potential collides with the ascending action potential. $a 6$ A synchronized response from the $3 \mathrm{MNs}$ that were initially excited by the transcranial stimulus is recorded as the second main negative deflection of the TSTtest trace. b In $\mathrm{TST}_{\text {control }}: b 1$ a maximal stimulus is applied at Erb's point. $b 2$ On all four MNs action potentials descend. $b 3$ After a delay, a maximal stimulus is applied at the wrist. $b 4$ The orthodromic action potentials are recorded as the first negative deflection of the TST control trace, the antidromic action potentials collide with the action potentials of the first stimulus at Erb's point. $b 5$ After a delay a maximal stimulus is applied at the Erb's point. $b 6$ A synchronized response from the $5 \mathrm{MNs}$ is recorded as the second negative deflection of the TST control trace. The test response is quantified as the ratio of

MEP amplitude ratio recorded from the left ADM (precontraction of $20 \%$ MVC, TMS intensity $150 \%$ RMT) was 66.3\% (SD 13.0) and from the right ADM 64.4\% (12.7). The average MEP area ratio was $107.9 \%$ (18.0) in the left and $106.3 \%$ (17.1) in the right ADM (Fig. 2a). MEP ampli-
(B) $\mathrm{TST}_{\text {control }}$

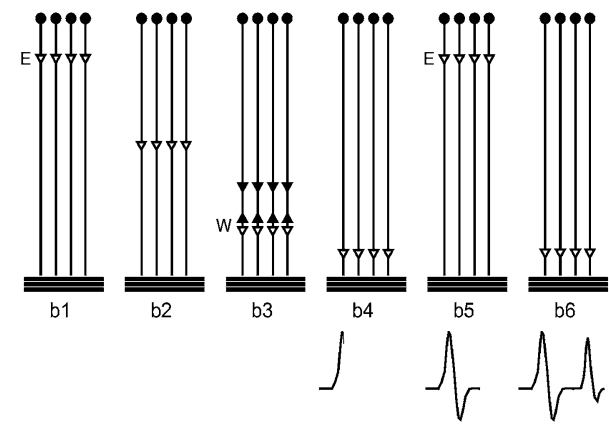

(D) QuadS control $_{\text {in }}$

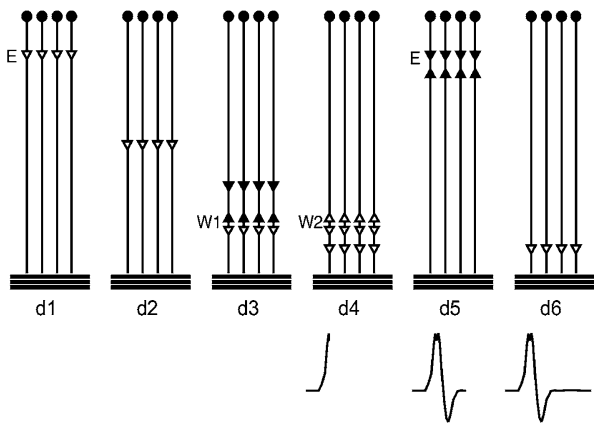

$\mathrm{TST}_{\text {test }}: \mathrm{TST}_{\text {control }}$ curves $(3 / 4=75 \%$ in this example). c Quadruple stimulation technique (QuadS) principle. $\mathbf{c}$ In QuadS $\mathrm{S}_{\text {test }}: c 1$ a submaximal transcranial stimulus excites 3 spinal MNs of 4 . $c 2$ On 3 of $4 \mathrm{MNs}$, TMS induced action potentials descend. Two of three spinal MNs are excited twice (MNs 1 and 2). c3 After a delay, a first maximal stimulus is applied at the wrist (W1). $c 4$ It gives rise to a first negative deflection of the recording trace. The antidromic action potentials collide with the first descending action potentials on MNs 1, 2, and 3. After $3 \mathrm{~ms}$ a second supramaximal stimulus is applied at the wrist (W2). The orthodromic action potentials give rise to a second negative deflection of the recording trace melting partially with the first one. The antidromic action potentials evoked by this stimulus collide with the repMNDs on MNs 1 and 2. The action potential on MNs 3 and 4 continue to ascend. c5 After an appropriate delay, a maximal stimulus is applied at the Erb's point. On MNs 3 and 4, the descending action potential collides with the ascending action potentials. $c 6$ A synchronized response from the $2 \mathrm{MNs}$ that conducted repMND is recorded as the second deflection of the QuadS $S_{\text {test }}$ trace. $\mathbf{d}$ In QuadS $\mathrm{S}_{\text {control }}: d l$ a maximal stimulus is applied at Erb's point. $d 2$ On $4 / 4$ neurons action potentials descend. $d 3$ After a delay, a maximal stimulus is applied to the wrist. $d 4$ It is recorded as the first deflection of the TST control trace. After a delay of $3 \mathrm{~ms}$, a second supramaximal stimulus is applied at the wrist. $d 5$ After an appropriate delay, a maximal stimulus is applied at Erb's point. The antidromic action potentials evoked by this stimulus collide with the second stimulus applied at the wrist. $d 6$ As a consequence no response is recorded in the control trace. The test response is quantified as the ratio of QuadS $\mathrm{test}_{\text {tes }}: \mathrm{TST}_{\text {control }}$ curves $(2 / 4=50 \%$ in this example)

tude and area ratios were not significantly different for the right and left ADM.

The average TST amplitude ratio in the left ADM was $88.1 \%$ (SD 8.4\%) and in the right ADM 89.8\% (9.8\%). The average TST area ratio was $87.5 \%(10.3 \%)$ in the left ADM 

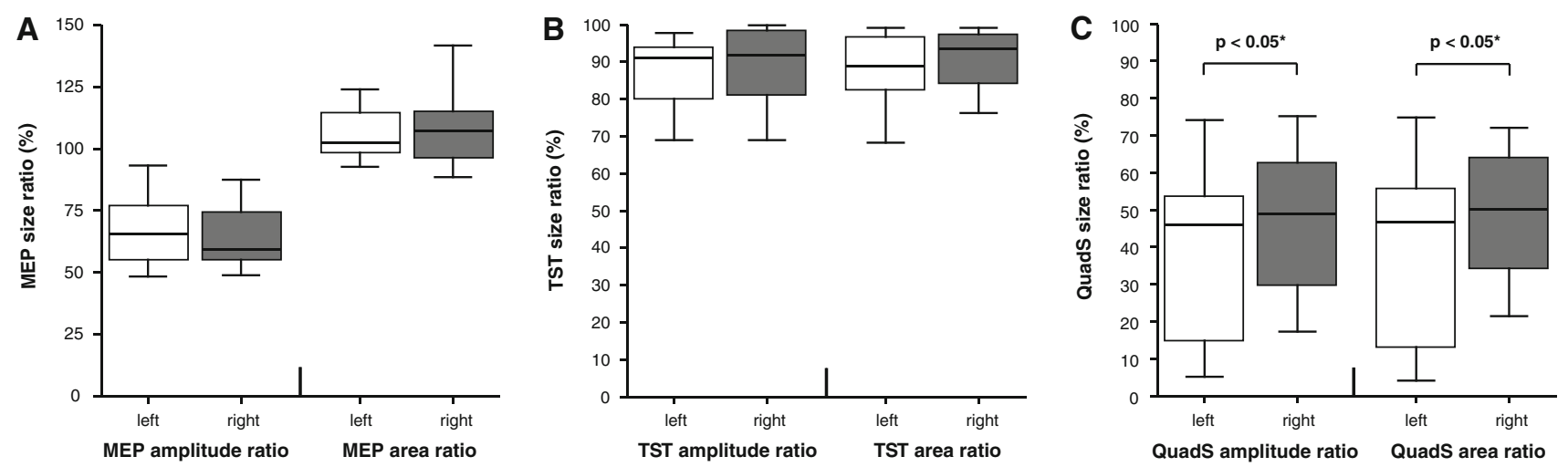

Fig. 2 Boxplots of MEP, TST and QuadS amplitude and area ratios of both hands $(n=20)$ : average MEP $(\mathbf{a})$ and TST $(\mathbf{b})$, amplitude and area ratios were not significantly different for the right and left hand. Both,
QuadS amplitude and area ratios were significantly larger in the dominant right hand (Wilcoxon test) (c)

\section{Precision movements}

The mean number of correctly performed taps during $15 \mathrm{~ms}$ was 35.6 (SD 4.2) for the left hand and 36.9 (SD 4.4) for the right hand $(P=0.015)$. The mean number of correct taps was greater for the right than for the left hand in 16 of the 20 subjects.

Assessment after training

RMT, MEP, and TST responses

Training did not affect RMT, MEP, and TST amplitude and area ratios of either hand (Table 1, Fig. 3).

\section{QuadS responses}

In the trained left ADM, the average QuadS amplitude and area ratios increased significantly after precision movement training (Kruskal-Wallis test: $\Delta$ QuadS amplitude ratio: $P=0.025 ; \Delta$ QuadS area ratio: $P=0.007$ ) (Table 1 , Fig. 3). No change occurred after force training. The

Table 1 Changes after training of precision or of force with the left hand

\begin{tabular}{|c|c|c|c|c|c|}
\hline \multirow{2}{*}{$\begin{array}{l}\text { Difference } \\
\text { (after training-before training) }\end{array}$} & \multicolumn{2}{|c|}{ Training of finger tapping $(n=8)$} & \multicolumn{2}{|c|}{ Training of force $(n=8)$} & \multirow{2}{*}{$\begin{array}{l}\text { Kruskal-Wallis tes } \\
P \text { values }\end{array}$} \\
\hline & Left hand & Right hand & Left hand & Right hand & \\
\hline$\Delta$ mean no. of precise taps (no./15 s) & $+9.8 \pm 4.4$ & $+1.3 \pm 3.4$ & $+2.4 \pm 4.3$ & $-0.5 \pm 4.2$ & $0.003 * *$ \\
\hline$\Delta \mathrm{RMT}(\%)$ & $+0.4 \pm 1.6$ & $+0.5 \pm 3.5$ & $-0.1 \pm 1.2$ & $-1.0 \pm 3.7$ & 0.76 \\
\hline$\Delta$ mean MEP amplitude ratio $(\%)$ & $-0.6 \pm 10.0$ & $-4.4 \pm 8.7$ & $-0.7 \pm 8.5$ & $+7.1 \pm 14.8$ & 0.46 \\
\hline$\Delta$ mean MEP area ratio $(\%)$ & $-6.3 \pm 12.7$ & $-3.8 \pm 13.1$ & $-5.0 \pm 10.5$ & $-0.2 \pm 12.2$ & 0.69 \\
\hline$\Delta$ mean TST amplitude ratio $(\%)$ & $+6.9 \pm 8.6$ & $+3.4 \pm 9.0$ & $+1.2 \pm 5.6$ & $-2.5 \pm 9.7$ & 0.27 \\
\hline$\Delta$ mean TST area ratio $(\%)$ & $+1.6 \pm 10.6$ & $+2.3 \pm 12.0$ & $+4.0 \pm 10.7$ & $-1.4 \pm 9.4$ & 0.95 \\
\hline$\Delta$ mean QuadS amplitude ratio $(\%)$ & $+12.4 \pm 16.2$ & $-6.9 \pm 16.1$ & $+1.9 \pm 10.6$ & $-10.5 \pm 13.0$ & $0.025^{*}$ \\
\hline$\Delta$ mean QuadS area ratio $(\%)$ & $+11.3 \pm 12.7$ & $-7.5 \pm 13.5$ & $+0.8 \pm 8.2$ & $-10.0 \pm 10.5$ & $0.007 * *$ \\
\hline
\end{tabular}

Values are shown \pm SD 

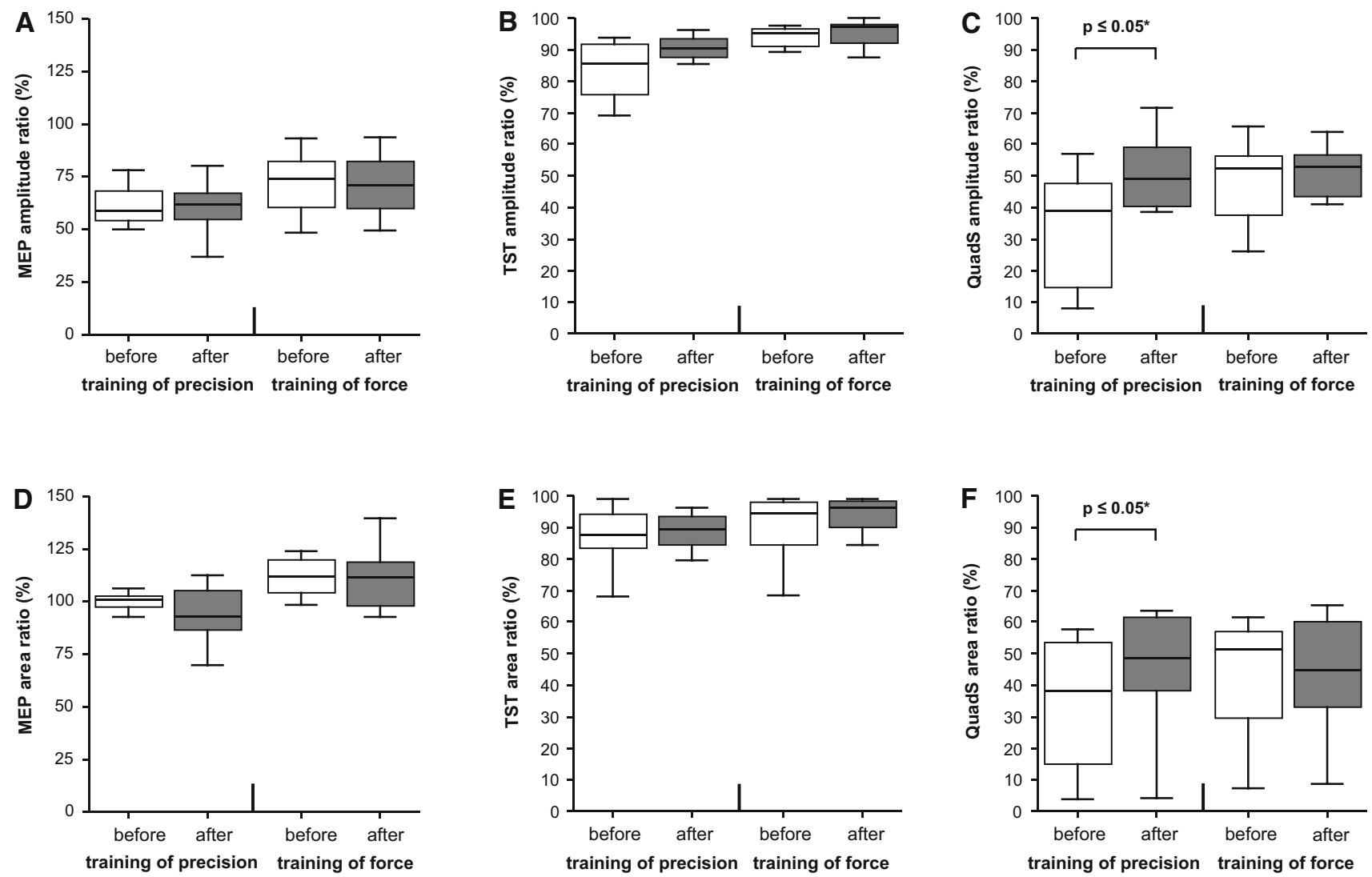

Fig. 3 Boxplots showing MEP, TST, and QuadS amplitude and area ratios of the left hand before and after training of either precision movements or force. MEP amplitude (a) and area (d) ratios remained unchanged by training. Training had also no effect on TST amplitude

performance of the right hand was not affected significantly by training of the left hand, independent of the training regimen (Table 1). However, there was a tendency to decreased QuadS responses after training independent of the training modality. This finding might reflect a training induced change in the balance of the inter-hemispheric inhibition.

\section{Precision movements}

The mean number of correct taps for the left hand increased from 33.4 to 44.5 (mean increase 9.8 , SD $4.4, P=0.003$ ) after training of precision movements, whereas no change occurred in the untrained, right hand. After force training of the left hand, the number of correct taps remained unchanged.

There was a significant positive correlation between the training induced increase of correct finger taps and the increase of the QuadS amplitude $\left(R^{2}=0.60, P=0.024\right)$ and area ratios $\left(R^{2}=0.54, P=0.038\right)$ (Fig. 4). A similar correlation was not found after force training and also not for the untrained right hand. (b) and area (e) ratios. QuadS amplitude (c) and area (f) ratios increased significantly after training of precision movements (Wilcoxon test) but not after training of force

\section{F-wave responses}

Ulnar F-wave studies were performed in eight subjects before and after training (four subjects each undergoing precision movement training and force training). After force training, the average ulnar F-wave areas increased in all four subjects whereas no changes were observed after precision movement training of the left hand or in the untrained right hand (Kruskal-Wallis test, $P=0.039$ ).

\section{Discussion}

After a single brain stimulus, spinal MNs may discharge more than once (Berardelli et al. 1991; Day et al. 1987; Hess et al. 1987; Naka and Mills 2000). The aim of the present study was to investigate the occurrence of such repMNDs compared with other parameters of cortico-motoneuronal excitability. We first compared stimuli given to the dominant versus the non-dominant hemisphere, since previous studies had indicated a greater excitability to 
Fig. 4 Dots represent the change in QuadsS amplitude (a) and QuadS area ratios (b) of the left hand in relation to the change in the precision test of Dig V. There was a significant positive correlation between the training induced increase of the number of correct finger taps and the increase of the QuadS amplitude and area ratios
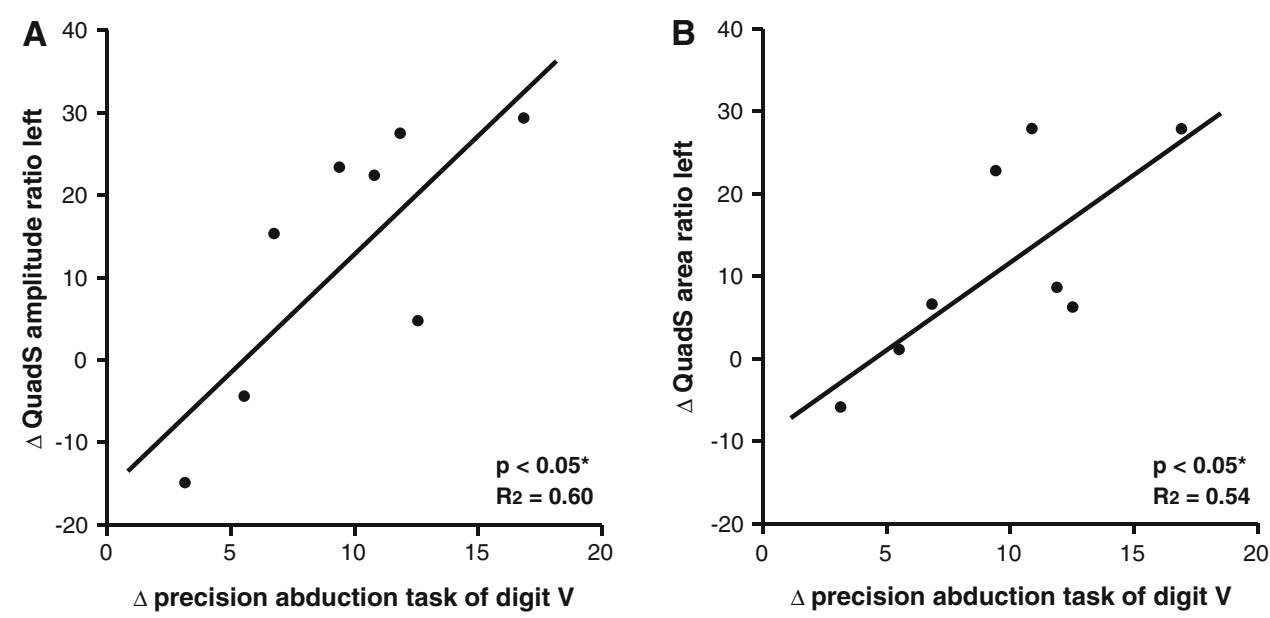

magnetic stimulation of the dominant hemisphere (Brouwer et al. 2001; Cantello et al. 1992; Civardi et al. 2000; De Gennaro et al. 2004; Krings et al. 1997; Netz et al. 1995; Triggs et al. 1999). We then attempted to actively manipulate the excitability of the cortico-motoneuronal pathway by two training regimens. Training of precision movements was previously shown to induce adaptations at the cortical level, whereas force training induces adaptations mainly at the spinal and muscular level (Carroll et al. 2002; Classen et al. 1998; Jensen et al. 2005; Pascual-Leone et al. 1995; Perez et al. 2004; Remple et al. 2001).

Our data demonstrate a differential behavior of the electrophysiological excitability measures in these situations. RepMNDs, in particular, appeared to be related to supraspinal, probably cortical excitability.

The TST eliminates influences of MN discharge desynchronization and of repMNDs on the response size (discussed in detail by Bühler et al. 2001; Magistris et al. 1998; Rösler et al. 2002). It is thus well suited to quantify the first MN discharges evoked by the brain stimulus. With the stimulation parameters chosen here (well above threshold, with ample facilitatory background contraction) some 80$90 \%$ of the MNs were brought to discharge at least once (Fig. 2b). Hence, changes of the TST response size in the present setting would indicate excitability changes of the highest threshold MNs, which are recruited last. No such changes were observed in the present experiments, and TST response size did not differ between the hemispheres. On the other hand, RMT assesses excitation of the first few MNs recruited by the brain stimulus (termed "core motor neurons"), which have the highest excitability. RMT did not differ between the hemispheres in our subjects and did not change with either of the training protocols (Table 1). Thus, the excitability of the lowest threshold MNs remained unchanged as well. Taken together, this suggests that the recruitment curve of first discharges (i.e., the "input-output curve") was not affected by the paradigms studied here. F-waves can serve as a measure of spinal motorneuronal excitability (Espiritu et al. 2003; Lin and Floeter 2004; Mercuri et al. 1996; Taniguchi et al. 2007). They remained unchanged by the precision training protocol, but increased by force training. The latter was expected since force training was shown to affect the excitability of spinal MNs (Carroll et al. 2002; Jensen et al. 2005).

The present investigation suggests an association between handedness and the skill for precision movements and the threshold for repMNDs. Our right-handed subjects performed significantly better with their dominant right hands in the precision movement task and repMNDs were more frequent in the recordings from the right hand (Fig. 2). Both, skill for precision movements and repMNDs increased with precision movement training (Table 1, Fig. 3). After the training, both, precision performance and repMNDs of the left hand increased to the levels previously measured on the right side. There was a positive linear correlation between the increase in QuadS size ratios and the increase in precision movement performance (Fig. 4a, b). Hence, it is highly probable that the origin of the lowered threshold for repMNDs observed here was supraspinal. First, handedness is determined by an asymmetry of the motor cortex rather than by spinal asymmetry (Brouwer et al. 2001; De Gennaro et al. 2004; Netz et al. 1995). Second, it has been demonstrated that skills training affects the size of MEPs and the cortical area of representation (Classen et al. 1998; Pascual-Leone et al. 1995; Perez et al. 2004). Third, our F-wave analysis did not reveal an effect on segmental spinal excitability induced by the precision movement training.

Therefore, we conclude that repMNDs in the present setting can serve as a measure of supraspinal and probably cortical excitability.

It was previously shown that MEPs elicited by stimulation of the dominant hemisphere were larger than those after stimulation of the non-dominant hemisphere (Brouwer 
et al. 2001; De Gennaro et al. 2004). In the present study we could not repeat this finding, probably due to the smaller number of subjects. The size of a MEP is determined by three factors, namely (i) the number of first corticospinal discharges, (ii) the desynchronization of the TMSinduced MN discharges and phase cancellation (Magistris et al. 1998), and (iii) by the number of repMNDs (Z'Graggen et al. 2005). The effect of these three factors can be sorted out with the present study. Our TST results indicate that the number of first MN discharges was similar for both hemispheres. In contrast, the number of repMNDs was larger after stimulation of the dominant hemisphere.

In summary, the results of the present study demonstrate that repMNDs are a parameter for cortico-spinal excitability, independent of the established measures (TST amplitude and area, RMT). The study suggests an association of repMNDs with handedness and the skill for precision movements. From a physiological view, a lowered threshold of the cerebral circuitry involved in precision movements might serve to enhance the properties of the system for finely tuned responses to the functional demands imposed on the motor system. Central plasticity enables the system to adapt to increased needs for precision movements.

Acknowledgments This study was supported by the Swiss National Science foundation (Grant 3200B0-100701). A.M.H. was supported by a Swiss national grant for a fellowship in clinical neurophysiology (Grant 31-226).

\section{References}

Arányi Z, Mathis J, Hess CW, Rösler KM (1998) Task-dependent facilitation of motor evoked potentials during dynamic and steady muscle contractions. Muscle Nerve 21:1309-1316

Berardelli A, Inghilleri M, Rothwell JC, Cruccu G, Manfredi M (1991) Multiple firing of motoneurones is produced by cortical stimulation but not by direct activation of descending motor tracts. Electroencephalogr Clin Neurophysiol 81:240-242

Brouwer B, Sale MV, Nordstrom MA (2001) Asymmetry of motor cortex excitability during a simple motor task: relationships with handedness and manual performance. Exp Brain Res 138:467-476

Bühler R, Magistris MR, Truffert A, Hess CW, Rösler KM (2001) The triple stimulation technique to study central motor conduction to the lower limbs. Clin Neurophysiol 112:938-949

Cantello R, Gianelli M, Civardi C, Mutani R (1992) Magnetic brain stimulation: the silent period after the motor evoked potential. Neurology 42:1951-1959

Carroll TJ, Riek S, Carson RG (2002) The sites of neural adaptation induced by resistance training in humans. J Physiol 544:641-652

Civardi C, Cavalli A, Naldi P, Varrasi C, Cantello R (2000) Hemispheric asymmetries of cortico-cortical connections in human hand motor areas. Clin Neurophysiol 111:624-629

Classen J, Liepert J, Wise SP, Hallett M, Cohen LG (1998) Rapid plasticity of human cortical movement representation induced by practice. J Neurophysiol 79:1117-1123

Conforto AB, Z'Graggen WJ, Kohl AS, Rösler KM, Kaelin-Lang A (2004) Impact of coil position and electrophysiological monitor- ing on determination of motor thresholds to transcranial magnetic stimulation. Clin Neurophysiol 115:812-819

Day BL, Rothwell JC, Thompson PD, Dick JP, Cowan JM, Berardelli A, Marsden CD (1987) Motor cortex stimulation in intact man. 2. Multiple descending volleys. Brain 110:1191-1209

De Gennaro L, Cristiani R, Bertini M, Curcio G, Ferrara M, Fratello F, Romei V, Rossini PM (2004) Handedness is mainly associated with an asymmetry of corticospinal excitability and not of transcallosal inhibition. Clin Neurophysiol 115:1305-1312

Espiritu MG, Lin CS, Burke D (2003) Motoneuron excitability and the F wave. Muscle Nerve 27:720-727

Hess CW, Mills KR, Murray NM (1987) Responses in small hand muscles from magnetic stimulation of the human brain. J Physiol 388:397-419

Jensen JL, Marstrand PC, Nielsen JB (2005) Motor skill training and strength training are associated with different plastic changes in the central nervous system. J Appl Physiol 99:1558-1568

Krings T, Buchbinder BR, Butler WE, Chiappa KH, Jiang HJ, Cosgrove GR, Rosen BR (1997) Functional magnetic resonance imaging and transcranial magnetic stimulation: complementary approaches in the evaluation of cortical motor function. Neurology 48:1406-1416

Lin JZ, Floeter MK (2004) Do F-wave measurements detect changes in motor neuron excitability? Muscle Nerve 30:289-294

Magistris MR, Rösler KM, Truffert A, Myers JP (1998) Transcranial stimulation excites virtually all motor neurons supplying the target muscle. A demonstration and a method improving the study of motor evoked potentials. Brain 121:437-450

Magistris MR, Rösler KM, Truffert A, Landis T, Hess CW (1999) A clinical study of motor evoked potentials using a triple stimulation technique. Brain 122:265-279

Mercuri B, Wassermann EM, Manganotti P, Ikoma K, Samii A, Hallett M (1996) Cortical modulation of spinal excitability: an F-wave study. Electroencephalogr Clin Neurophysiol 101:16-24

Muellbacher W, Ziemann U, Boroojerdi B, Cohen L, Hallett M (2001) Role of the human motor cortex in rapid motor learning. Exp Brain Res 136:431-438

Naka D, Mills KR (2000) Further evidence for corticomotor hyperexcitability in amyotrophic lateral sclerosis. Muscle Nerve 23:1044-1050

Netz J, Ziemann U, Homberg V (1995) Hemispheric asymmetry of transcallosal inhibition in man. Exp Brain Res 104:527-533

Oldfield RC (1971) The assessment and analysis of handedness: the Edinburgh inventory. Neuropsychologia 9:97-113

Pascual-Leone A, Nguyet D, Cohen LG, Brasil-Neto JP, Cammarota A, Hallett M (1995) Modulation of muscle responses evoked by transcranial magnetic stimulation during the acquisition of new fine motor skills. J Neurophysiol 74:1037-1045

Perez MA, Lungholt BK, Nyborg K, Nielsen JB (2004) Motor skill training induces changes in the excitability of the leg cortical area in healthy humans. Exp Brain Res 159:197-205

Remple MS, Bruneau RM, VandenBerg PM, Goertzen C, Kleim JA (2001) Sensitivity of cortical movement representations to motor experience: evidence that skill learning but not strength training induces cortical reorganization. Behav Brain Res 123:133-141

Rösler KM, Petrow E, Mathis J, Aranyi Z, Hess CW, Magistris MR (2002) Effect of discharge desynchronization on the size of motor evoked potentials: an analysis. Clin Neurophysiol 113:1680 1687

Rossini PM, Barker AT, Berardelli A, Caramia MD, Caruso G, Cracco RQ, Dimitrijevic MR, Hallett M, Katayama Y, Lucking CH et al (1994) Non-invasive electrical and magnetic stimulation of the brain, spinal cord and roots: basic principles and procedures for routine clinical application. Report of an IFCN committee. Electroencephalogr Clin Neurophysiol 91:79-92 
Roth G, Magistris MR (1987) Detection of conduction block by monopolar percutaneous stimulation of the brachial plexus. Electromyogr Clin Neurophysiol 27:45-53

Taniguchi S, Kimura J, Yanagisawa T, Okada F, Yamada T, Ootsuka $\mathrm{T}$ (2007) Rest-induced suppression of anterior horn cell excitability as measured by F waves: comparison between volitionally inactivated and control muscles. Muscle Nerve 37:343-349
Triggs WJ, Subramanium B, Rossi F (1999) Hand preference and transcranial magnetic stimulation asymmetry of cortical motor representation. Brain Res 835:324-329

Z'Graggen WJ, Humm AM, Durisch N, Magistris MR, Rösler KM (2005) Repetitive spinal motor neuron discharges following single transcranial magnetic stimuli: a quantitative study. Clin Neurophysiol 116:1628-1637 